\title{
Positional differences in the cardiorespiratory, autonomic, and somatic profiles of professional soccer players
}

\author{
Martin Semjon $^{1}$, Michal Botek ${ }^{1, *}$, Zbyněk Svozil ${ }^{1}$, and Andrew J. McKune ${ }^{2,3}$ \\ ${ }^{1}$ Faculty of Physical Culture, Palacký University Olomouc, Olomouc, Czech Republic; ${ }^{2}$ Discipline of Sport and Exercise Sci- \\ ence, Faculty of Health, Research Institute for Sport and Exercise, University of Canberra, Canberra, Australia; and ${ }^{3}$ Disci- \\ pline of Biokinetics, Exercise and Leisure Sciences, School of Health, University of KwaZulu-Natal, Durban, South Africa
}

Copyright: (C) 2016 M. Semjon et al. This is an open access article licensed under the Creative Commons Attribution License (http://creativecommons.org/licenses/by/4.0/).

Background: In order to optimize training in soccer, knowledge about the specific position fitness demands and characteristics is required. Objective: The aim of the study was to determine whether there are position specific differences in somatic, cardiorespiratory and autonomic cardiac profiles of professional senior Czech soccer players. Methods: All players $(N=120)$ were divided into six groups according to field positions: goalkeeper (GK; $n=11)$, external defenders (ED; $n=15)$, central defenders (CD; $n=18)$, external midfielders (EM; $n=18)$, central midfielders $(\mathrm{CM} ; n=24)$ and forwards $(\mathrm{F} ; n=34)$. Players underwent anthropometrical and heart rate variability (HRV) assessment, and a maximal incremental running test in order to obtain maximal oxygen uptake $\left(\mathrm{VO}_{2}\right.$ max $)$ and heart rate. HRV variables were transformed using the natural logarithm (Ln). Results: GK and CD were significantly $(p=.005)$ heavier than ED, EM, CM, and F; while F were significantly $(p=.026)$ heavier than CM and EM. GK and CD were significantly $(p=.008)$ taller than ED, EM, CM, F; and EM were significantly $(p=.041)$ shorter than CM and F. The only significant $(p=.043)$ difference in percentage of body fat was observed in CD compared with CM. A significantly ( $p=.021)$ lower $\mathrm{VO}_{2}$ max was observed for GK compared with ED, CD, EM, CM, and F. Supine HRV was significantly $(p=.039)$ lower for Ln LF/HF in GK compared with F. Standing HRV was significantly $(p=.03)$ lower for Ln LF in CD compared with both ED and F, significantly $(p=.028)$ higher for Ln LF/HF in ED compared with $\mathrm{CD}$ and EM. Conclusions: In soccer, specific positions are associated with different height, body mass and aerobic capacity. A lower $\mathrm{VO}_{2}$ max and vagal activity in GK compared with other playing positions may not be considered as disadvantage for performance in this specific playing position.

Keywords: maximal oxygen uptake, vagal activity, body composition, heart rate variability

\section{Introduction}

Elite performance in soccer represents a composite of high level physical performance characteristics that, in turn, depend upon a variety of anthropometrical and physiological properties, as well as on the training and health status of the individual athlete (MacArthur \& North, 2005). In soccer, is well known that different field positions require specific fitness characteristics (Magalhães Saleset al., 2014; Stølen, Chamari, Castagna, \& Wisløff, 2005; Wisløff, Helgerud, \& Hoff, 1998). Therefore, identifying each player's specialized

\footnotetext{
* Address for correspondence: Michal Botek, Department of Natural Sciences in Kinanthropology, Faculty of Physical Culture, Palacký University Olomouc, třída Míru 117, 77111 Olomouc, Czech Republic. E-mail: michal.botek@upol.cz
}

position has been suggested to be important. Such a process will help optimize their physical, physiological, psychological, technical and tactical development in order to prepare them for higher playing levels later in their career (Gil, Gil, Ruiz, Irazusta, \& Irazusta, 2007; Strøyer, Hansen, \& Klausen, 2004).

Soccer is an aerobic sport that also places demands on the anaerobic energy system for speed endurance, strength, speed and explosiveness (dos Santos et al., 2014; Strøyer et al., 2004). $\mathrm{VO}_{2} \max$ values for professional soccer players that play in the Champions League range between $60-65 \mathrm{ml} \cdot \mathrm{kg}^{-1} \cdot \mathrm{min}^{-1}$ (Helgerud, Rodas, Kemi, \& Hoff, 2011).

From different playing position point of view, central defenders $(C D)$ spend significantly more time walking and jogging $\left(0-11 \mathrm{~km} \cdot \mathrm{h}^{-1}\right)$ during matches and also spend the least time in all other work-intensities 
and cover the least distance (Di Salvo et al., 2007). In contrast, midfield players, such as central midfielders (CM) and external midfielders (EM), spend the shortest amount of time walking and jogging, but covered the most distance at low and moderate running speeds. EM spent the most amount of time and cover the most distance at high-running speeds and sprinting (Rampinini, Sassi, Sassi, \& Impellizzeri, 2004).

Body composition, anthropometric dimensions, and morphological characteristics play vital roles in determining the success of a soccer player (Keogh, 1999; Silvestre, West, Maresh, \& Kraemer, 2006). Accurate evaluation of these parameters is required to identify specific characteristics that are a necessity for optimal soccer performance which in turn may influence player selection (Gil, Ruiz, Irazusta, Gil, \& Irazusta, 2007). Authors investigating body fat (BF) in different field positions in soccer (Reilly, 1996) found small differences in percent BF among the outfield positions, although midfielders tended to have lower BF levels.

Spectral analysis (SA) of RR intervals to determine heart rate variability (HRV) is accepted as a non-invasive method for autonomic cardiac regulation assessment (Akselrod et al., 1981). It is well known that orthostatic stress decreases vagal activity and stimulates sympathetic activity (Eckberg, 1997; Malliani, Pagani, Lombardi, \& Cerutti, 1991), whereas clinostasis stimulates vagal activity (Task Force of the European Society of Cardiology and the North American Society of Pacing and Electrophysiology, 1996). Therefore, to enhance the sensitivity of measuring small reciprocal changes in the vagal and sympathetic pathways in elite athletes it has been suggested that HRV should be quantified from electrocardiogram (ECG) sampling in both standing and supine positions (Botek, McKune, Krejčí, Stejskal, \& Gába, 2014).

Endurance training has been shown to induce a decline in resting heart rate $\left(\mathrm{HR}_{\text {rest }}\right)$, as well as increase overall resting HRV as a result of elevated parasympathetic (vagal) activity (Aubert, Seps, \& Beckers, 2003; Carter, Banister, \& Blaber, 2003; Hedelin, Wiklund, Bjerle, \& Henriksson-Larsén, 2000; Kouidi, Haritonidis, Koutlianos, \& Deligiannis, 2002). Vagal activity dominance allows athletes to recover faster and accelerate the transition from catabolism to anabolism (Hautala et al., 2001).

Both, high frequency (HF) oscillations in RR intervals (Pichot et al., 2000), and a root of the mean square of successive differences (Buchheit, 2014) have been suggested to reflect the cardiac vagal outflow, while reciprocal changes between vagal and sympathetic activity are evaluated via analysis of low frequency power (LF) (Task Force of the European Society of
Cardiology and the North American Society of Pacing and Electrophysiology, 1996) and LF/HF (Ori, Monir, Weiss, Sayhouni, \& Singer, 1992), respectively.

The main aim of this study was to assess whether different playing positions in soccer require specific somatic, aerobic and HRV characteristics among Czech professional soccer players.

\section{Methods}

\section{Participants}

This study included 164 soccer players from clubs that play in the first division of the senior Czech Republic Soccer League. These players had undergone preseason laboratory testing between 2009 and 2014. One hundred and twenty players performed all the preseason measurements (anthropometrical evaluation, HRV, maximal incremental running test to determine maximal aerobic capacity), while 44 players only performed the incremental running test, and were therefore included for the cardiorespiratory performance analysis only.

Inclusion criteria for participation in this study were the following - each player should: 1) participate in the entire laboratory testing protocol; 2) be free of any health complications that may limit performance in any laboratory test, especially the maximal incremental running test; 3 ) be a member of the senior team squad at the time of testing. The study was approved by the Ethics Committee of the Faculty of Physical Culture, Palacký University Olomouc. All of the subjects participating in the study were volunteers and had given their written informed consent.

\section{Testing procedures}

The entire testing protocol was performed between 8-12 a.m. on a single day in following order - a resting autonomic cardiac activity assessment; basic anthropological measurement; and maximal incremental running test. For the focus of the current paper, only age and data related to anthropometry, autonomic cardiac activity and aerobic endurance capacity were included.

\section{Autonomic cardiac activity assessment}

The monitoring of autonomic cardiac activity was performed between 8-12 a.m. in a laboratory where the ambient temperature ranged from 22 to $24^{\circ} \mathrm{C}$. During the measurement, each player was shielded against acoustic and visual disturbances. To determine the resting HR and HRV variables, the ECG signal was measured at a sampling frequency of $1000 \mathrm{~Hz}$ using VarCor PF7 (DIMEA Group, Olomouc, Czech Republic). The ECG sampling was performed during an 
orthoclinostatics maneuver (supine-standing-supine) up to January 2013, and in the following period, a timemodified orthoclinostatics test was used, because both orthoclinostatics challenges provide comparable HRV results (Botek, Krejčí, Neuls, \& Novotný, 2013).

The ECG record was examined and all premature ventricular contractions, missing beats and any artefacts, were manually filtered. A set of 300 artefactfree subsequent RR intervals was obtained from each phase. Spectral analysis of HRV was used to assess the autonomic cardiac activity and was performed using the Fast Fourier Transform. Two frequency bands were analyzed - the HF power $(0.15$ to $0.50 \mathrm{~Hz})$ that is thought to represent respiratory related cardiac vagal activity (Task Force of the European Society of Cardiology and the North American Society of Pacing and Electrophysiology, 1996); and the LF power (0.05 to $0.15 \mathrm{~Hz}$ ) that is considered to exhibit baroreflex activity together with sympathetic and vagal outflow (Task Force of the European Society of Cardiology and the North American Society of Pacing and Electrophysiology, 1996) whereas the LF/HF ratio reflects the sympathovagal balance (Ori et al., 1992). A time domain variable, the root mean square of successive difference in RR intervals (rMSSD) was also used. rMSSD is regarded as an index of vagal activity (Buchheit, 2014) that is thought to be resistant to the effects of breathing frequency (Penttilä et al., 2001).

\section{Anthropometrical measurement}

The soccer players had their body height and body mass measured using the SOEHNLE 7307 (Leifheit, Nassau, Germany). The \% BF was determined using bioimpedance analysis (Tanita BC-418 MA, Tanita, Tokyo, Japan).

\section{Maximal incremental running test}

Each soccer player underwent a graded maximal stress test on a treadmill (Lode Valiant, Lode, Groningen, Netherlands) in order to obtain $\mathrm{VO}_{2} \max$ and $\mathrm{HR}_{\max }$. The exercise protocol consisted of a 4 minute warm-up period ( 2 minutes at $8 \mathrm{~km} \cdot \mathrm{h}^{-1}$ with a $0 \%$ inclination and a further 2 minutes at the same speed with a $5 \%$ inclination). The speed was then increased to $10 \mathrm{~km} \cdot \mathrm{h}^{-1}$ for 1 minute with the gradient kept at $5 \%$. For each minute thereafter speed increased by $1 \mathrm{~km} \cdot \mathrm{h}^{-1}$ with the gradient at $5 \%$ up to maximal speed of $16 \mathrm{~km} \cdot \mathrm{h}^{-1}$. From this stage only inclination increased by $2.5 \%$ every minute until exhaustion. Breath-by-breath ventilation and gas exchange were continuously analyzed (Ergostik, Geratherm Respiratory, Bad Kissingen, Germany) during the exercise with the data averaged to 30 seconds for analysis. The following criteria were used to document that $\mathrm{VO}_{2} \max$ was achieved: 1) a lack of increase in $\mathrm{VO}_{2}$ upon an increase in work rate, 2) respiratory exchange ratio > 1.10 (Shephard \& Åstrand, 1992) and 3) a $\mathrm{HR}_{\text {max }}$ higher than $85 \%$ of age-predicted value $\left(\mathrm{HR}_{\max }=220-\right.$ age $)$. $\mathrm{VO}_{2}$ max was recorded as the highest oxygen consumption value in the final 30 seconds of the test. HR responses (Polar Wind Link+PolarH3, Polar, Kempele, Finland) were monitored continuously during the maximal stress test.

\section{Statistical analysis}

The subjects $(N=120)$ were divided into six groups according to field position: GK $(n=11), \mathrm{ED}(n=15)$, $\mathrm{CD}(n=18), \mathrm{EM}(n=18), \mathrm{CM}(n=24)$, and $\mathrm{F}$ $(n=34)$. A one-way analysis of variance (ANOVA) with Fisher's LSD post-hoc test was used to evaluate the differences between player positions. Due to the HRV variables demonstrating abnormal distribution, a natural logarithm (Ln) was applied. After Ln-transformation, all HRV variables were normally distributed. Statistical analyses were performed using STATISTICA (Version 12.0; StatSoft, Tulsa, OK, USA) with $p<.05$ considered statistically significant.

\section{Results}

Player descriptive statistics and ANOVA results for anthropometric, aerobic capacity and HRV variables are shown in Table 1 and 2. Based on the ANOVA result (Table 1) there was a significant age difference ( $p=.025$ ) between $\mathrm{CD}$ and F. GK and CD were significantly $(p=.005)$ heavier than ED, EM, CM, and $\mathrm{F}$; while $\mathrm{F}$ were significantly $(p=.026)$ heavier than CM and EM. GK and CD were significantly ( $p=.008)$ taller than ED, EM, CM, F; and EM were significantly ( $p=.041$ ) shorter than CM and F. The only significant ( $p=.043$ ) difference in \% BF was observed in CD compared with CM.

Based on the ANOVA result (Table 1), $\mathrm{VO}_{2} \max$ $\left(\mathrm{ml} \cdot \mathrm{kg}^{-1} \cdot \mathrm{min}^{-1}\right)$ was significantly $(p=.021)$ lower for GK compared with ED, CD, EM, CM, F. There were no significant differences in $\mathrm{HR}_{\text {rest }}\left(\mathrm{b} \cdot \mathrm{min}^{-1}\right)$ among all groups.

Spectral analysis of HRV was assessed in the standing and supine positions. Based on the ANOVA results (Table 2), there were significant differences between the playing positions for a few HRV variables. Standing LnLF was significantly ( $p=.030)$ lower for CD compared with ED and F. Standing Ln LF/HF was significantly ( $p=.028$ ) higher for ED compared with CD and EM. GK exhibited significantly $(p=.039)$ lower supine Ln rMSSD compared with F. 
Table 1

Anthropometric and cardiorespiratory values according to playing positions (mean $\pm S D$ )

\begin{tabular}{|c|c|c|c|c|c|c|}
\hline & GK & ED & $\mathrm{CD}$ & EM & $\mathrm{CM}$ & $\mathrm{F}$ \\
\hline \multicolumn{7}{|l|}{ Part $1(N=120)$} \\
\hline$n$ & 11 & 15 & 18 & 18 & 24 & 34 \\
\hline Age (years) & $26.6 \pm 6.5$ & $26.7 \pm 4.8$ & $27.3 \pm 6.2^{\mathrm{a}}$ & $25.3 \pm 4.2$ & $25.8 \pm 5.3$ & $24.0 \pm 3.6$ \\
\hline Body mass (kg) & $87.0 \pm 4.6^{\mathrm{b}}$ & $76.5 \pm 4.8$ & $84.5 \pm 4.6^{\mathrm{b}}$ & $74.6 \pm 5.1$ & $75.8 \pm 6.3$ & $79.4 \pm 7.7 \mathrm{c}$ \\
\hline Body height $(\mathrm{cm})$ & $188.6 \pm 3.3^{\mathrm{d}}$ & $180.7 \pm 3.6$ & $187.9 \pm 4.9^{\mathrm{d}}$ & $177.6 \pm 4.4^{\mathrm{e}}$ & $181.2 \pm 5.8$ & $183.3 \pm 7.2$ \\
\hline Body fat $(\%)$ & $11.5 \pm 3.1$ & $11.2 \pm 2.4$ & $11.7 \pm 2.8^{f}$ & $10.3 \pm 2.1$ & $9.9 \pm 2.9$ & $10.4 \pm 3.1$ \\
\hline \multicolumn{7}{|l|}{ Part $2(N=164)$} \\
\hline$n$ & 21 & 20 & 23 & 23 & 31 & 46 \\
\hline $\mathrm{HR}_{\text {rest }}\left(\mathrm{b} \cdot \mathrm{min}^{-1}\right)$ & $52.0 \pm 8.8$ & $51.4 \pm 6.5$ & $50.6 \pm 8.0$ & $52.6 \pm 7.5$ & $52.8 \pm 10.6$ & $51.1 \pm 7.5$ \\
\hline $\mathrm{VO}_{2} \max \left(\mathrm{ml} \cdot \mathrm{kg}^{-1} \cdot \mathrm{min}^{-1}\right)$ & $54.0 \pm 3.4^{\mathrm{g}}$ & $58.7 \pm 4.1$ & $58.0 \pm 6.1$ & $60.2 \pm 4.7$ & $59.3 \pm 4.5$ & $58.5 \pm 3.5$ \\
\hline
\end{tabular}

Note. $\mathrm{GK}=$ goalkeepers; $\mathrm{ED}=$ external defenders; $\mathrm{CD}=$ central defenders; $\mathrm{EM}=$ external midfielders; $\mathrm{CM}=$ central midfielders;

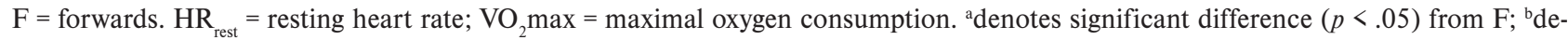
notes significant difference $(p<.05)$ from ED, EM, CM, F; ${ }^{\circ}$ denotes significant difference $(p<.05)$ from CM and EM; denotes significant difference $(p<.05)$ from ED, EM, CM, F; ${ }^{\mathrm{e} d e n o t e s ~ s i g n i f i c a n t ~ d i f f e r e n c e ~}(p<.05)$ from $\mathrm{CM}$ and $\mathrm{F}$; $\mathrm{f}$ denotes significant difference $(p<.05)$ from $\mathrm{CM}$; ${ }^{\mathrm{g}}$ denotes significant difference $(p<.05)$ from ED, CD, EM, CM, F.

Table 2

Values of SA HRV according to playing positions (mean $\pm S D ; N=120$ )

\begin{tabular}{|c|c|c|c|c|c|c|}
\hline & GK $(n=11)$ & $\mathrm{ED}(n=15)$ & $\mathrm{CD}(n=18)$ & $\mathrm{EM}(n=18)$ & $\mathrm{CM}(n=24)$ & $\mathrm{F}(n=34)$ \\
\hline \multicolumn{7}{|c|}{ Standing position in orthoclinostatic maneuver } \\
\hline $\operatorname{Ln} \operatorname{LF}\left(\mathrm{ms}^{2}\right)$ & $6.53 \pm 1.29$ & $7.07 \pm 0.80$ & $6.26 \pm 1.19^{\mathrm{a}}$ & $6.62 \pm 1.01$ & $6.79 \pm 0.77$ & $6.90 \pm 0.97$ \\
\hline Ln HF $\left(\mathrm{ms}^{2}\right)$ & $5.12 \pm 1.71$ & $5.12 \pm 1.29$ & $5.13 \pm 1.16$ & $5.42 \pm 1.20$ & $5.18 \pm 0.95$ & $5.39 \pm 1.13$ \\
\hline $\operatorname{Ln} \mathrm{LF} / \mathrm{HF}\left(\mathrm{ms}^{2}\right)$ & $1.40 \pm 1.62$ & $1.95 \pm 0.97^{\mathrm{b}}$ & $1.13 \pm 1.09$ & $1.20 \pm 0.93$ & $1.61 \pm 0.61$ & $1.51 \pm 0.83$ \\
\hline Ln rMSSD (ms) & $3.07 \pm 0.77$ & $3.31 \pm 0.51$ & $3.08 \pm 0.65$ & $3.23 \pm 0.49$ & $3.25 \pm 0.48$ & $3.28 \pm 0.58$ \\
\hline \multicolumn{7}{|c|}{ Supine position in orthoclinostatic maneuver } \\
\hline $\operatorname{Ln} \operatorname{LF}\left(\mathrm{ms}^{2}\right)$ & $6.44 \pm 1.05$ & $6.66 \pm 1.26$ & $6.42 \pm 1.09$ & $6.71 \pm 1.08$ & $6.14 \pm 0.98$ & $6.24 \pm 1.22$ \\
\hline Ln HF $\left(\mathrm{ms}^{2}\right)$ & $6.80 \pm 1.31$ & $7.37 \pm 0.89$ & $7.24 \pm 0.97$ & $7.49 \pm 1.03$ & $7.23 \pm 1.01$ & $7.40 \pm 0.97$ \\
\hline $\mathrm{Ln} \mathrm{LF} / \mathrm{HF}\left(\mathrm{ms}^{2}\right)$ & $-0.37 \pm 0.85$ & $-0.71 \pm 0.84$ & $-0.82 \pm 1.06$ & $-0.78 \pm 0.93$ & $-1.09 \pm 1.26$ & $-1.16 \pm 1.23$ \\
\hline Ln rMSSD (ms) & $4.12 \pm 0.64^{\mathrm{c}}$ & $4.43 \pm 0.45$ & $4.32 \pm 0.54$ & $4.42 \pm 0.56$ & $4.34 \pm 0.57$ & $4.44 \pm 0.47$ \\
\hline
\end{tabular}

Note. $\mathrm{GK}=$ goalkeepers; $\mathrm{ED}=$ external defenders; $\mathrm{CD}=$ central defenders; $\mathrm{EM}=$ external midfielders; $\mathrm{CM}=$ central midfielders; $\mathrm{F}=$ forwards. $\mathrm{Ln} \mathrm{LF}=$ natural logarithm of low-frequency power; $\mathrm{Ln} \mathrm{HF}=$ natural logarithm of high-frequency power; $\mathrm{Ln} \mathrm{LF} / \mathrm{HF}=$ natural logarithm of low-frequency/high-frequency ratio; Ln rMSSD = natural logarithm of root mean square successive difference of RR intervals. ${ }^{a}$ denotes significant difference $(p<.05)$ from ED and F; ${ }^{b}$ denotes significant difference $(p<.05)$ from CD and EM; ${ }^{\mathrm{c}}$ denotes significant difference $(p<.05)$ from $\mathrm{F}$.

\section{Discussion}

The present study is the first report the anthropometric, cardiorespiratory and HRV characteristics of professional players in the senior Czech Republic Soccer League. The study evaluated whether these variables differ amongst playing positions. The primary findings of this study were that a) GK and CD were significantly heavier and taller than ED, EM, CM, and F; b) there are no significant differences in cardiorespiratory performance and vagal activity among outfield players, and c) GK demonstrated a lower values of $\mathrm{VO}_{2} \max$ together with lower cardiac vagal activity compared with other playing positions.

In the present study, the players were divided into six different groups according to their field positions. Currently, the trend in world soccer is for an increase in average body height (Joksimović, Smajić, Molnar, \& Stanković, 2009). Compared with a number of other nations the Czech players are taller on average. Specifically, they are $5.4 \mathrm{~cm}$ taller than players in the United Arab Emirates elite League (Magalhães Sales et al., 2014), as well as taller than players in the Croatian League (Matković, Janković, Ružić, \& Leko, 2003) and 
the Northern Irish League (Wilson \& O'Donoghue, 2005). However, players from the English Premier League are of similar height (Milsom et al., 2015) to the Czech players. Unsurprisingly, relating to the specific requirements of their positions, the tallest and heaviest players in the present study were the GK and the CD (advantage in the air on defense), while the shortest were the EM (agility advantage). These results are supported by previous research (Di Salvo et al., 2007; Ekblom, 1986; Magalhães Sales et al., 2014; Matković et al., 2003; Rampinini et al., 2004; Milsom, et al., 2015; Sutton, Scott, Wallace, \& Reilly, 2009). There were also significant differences in body mass between the external and central positions. This result was in contrast to other studies where differences between the central and external positions were not found (Carling \& Orhant, 2010; Michailidis et al., 2013).

The mean of percentage in $\mathrm{BF}(\% \mathrm{BF})$ in the players was $10.7 \pm 2.8 \%$ that is similar to the values reported Le Gall, Carling, Williams, and Reilly (2010). In addition, we found a significant difference in \% BF only between $\mathrm{CD}$ and CM. Some researchers (Carling \& Orhant, 2010; Michailidis et al., 2013) reported a higher \% BF in EM compared with other positions.

Aerobic performance represented by $\mathrm{VO}_{2} \max$ is regarded as an important physiological parameter for optimal performance (Casajus, 2001). Elite soccer players have been reported to achieve values in the range of $55-70 \mathrm{ml} \cdot \mathrm{kg}^{-1} \cdot \mathrm{min}^{-1}$ (Stølen et al., 2005). In this study, there were no differences in $\mathrm{VO}_{2} \max$ between the outfield positions, however, the GK had significantly lower $\mathrm{VO}_{2}$ max values compared with the other positions. The difference in $\mathrm{VO}_{2}$ max between the GK and outfield players was in the range of 4.0 to $6.2 \mathrm{ml} \cdot \mathrm{kg}^{-1} \cdot \mathrm{min}^{-1}$. The difference could be explained by different training emphasis and match loads. In a previous study, compared with the present study, players achieved a $\mathrm{VO}_{2} \max$ that was $6.0 \mathrm{ml} \cdot \mathrm{kg}^{-1} \cdot \mathrm{min}^{-1}$ higher per position (Magalhães Sales et al., 2014). However, in contrary to our research, Magalhães Sales et al. (2014) obtained their $\mathrm{VO}_{2}$ max values indirectly based on an equation. Thus, we feel that a large discrepancy in $\mathrm{VO}_{2}$ max level between studies was due to methodological inconsistency. Although it has been recommended that players should have $\mathrm{VO}_{2}$ max values greater than $60 \mathrm{ml} \cdot \mathrm{min}^{-1} \cdot \mathrm{kg}^{-1}$ to be competitive at the highest level (Reilly, Bangsbo, \& Franks, 2000), in the present study, only the EM achieved this value. However, anaerobic power and capacity are also important in soccer (Stølen et al., 2005; Wisløff et al., 1998). Therefore $\mathrm{VO}_{2}$ max values should be viewed alongside speed, agility, power, and repeat sprint ability for a more appropriate assessment (Stølen et al., 2005; Wisløff et al., 1998).
Although we found no significant differences in vagal activity, represented by Ln HF (Aubert et al., 2003), the highest Ln HF was demonstrated in EM who also had the highest $\mathrm{VO}_{2} \max$ value. It has been reported that inter individual variation in adaptation to aerobic training may depend on baseline ANS activity level, particularly vagal activity (Hautala, Kiviniemi, \& Tulppo, 2009). Naranjo Orellana, de la Cruz Torres, Sarabia Cachadiña, de Hoyo, and Domínguez Cobo (2015) measured cardiac vagal activity (rMSSD) in Spanish elite soccer players and found lower vagal activity in both supine and standing compared with the present study. Research has suggested that increases in vagal-related HRV indices are generally associated with improved cardiorespiratory fitness (Botek, Krejčí, \& Weisser, 2014; Buchheit et al., 2008, 2010; Hautala et al., 2009; Hedelin, Bjerle, \& Henriksson-Larsén, 2001) and performance (Atlaoui et al., 2007; Botek et al., 2014; Lamberts \& Lambert, 2009; Lamberts, Swart, Capostagno, Noakes, \& Lambert, 2010), while decreases are associated with chronic fatigue and/or impaired physical performance (Borresen \& Lambert, 2008; Bosquet, Merkari, Arvisais, \& Aubert, 2008; Pichot et al., 2000). With regards to sympathovagal balance ( $\mathrm{Ln} \mathrm{LF} / \mathrm{HF}$ ) in this study, a significantly higher ratio was found in standing for ED compared with $\mathrm{EM}$ and $\mathrm{CD}$. In addition, $\mathrm{CD}$ demonstrated a significantly lower Ln LF compared with ED and F. GK demonstrated a significantly lower vagal activity compared with $\mathrm{F}$ in supine. The increase in vagal activity has been widely accepted to be endurance-related adaptation of ANS in endurance-trained athletes (Aubert et al., 2003). In fact, GK perform during their preparation/matches a lower amount of high intensity endurance exercises compared with other outfield players (Stølen et al., 2005), and therefore, we suggested that this aspect may lead to low vagal activity together with $\mathrm{VO}_{2}$ max level in our GK.

\section{Study limitations}

Limitations of the study include the lack of tests that examined other parameters important for soccer performance such as acceleration, speed, agility, repeatsprint, perceptual and cognitive ability. Also additional HRV measurements are needed to ensure a better indication of true HRV baseline.

\section{Conclusions}

To conclude, this study found that different playing positions require specific somatic characteristics, while aerobic capacity and HRV seems to be comparable amongst outfield playing positions. The somatic 
results are similar to those reported in previous studies performed on professional soccer players. However, aerobic capacity seems to be lower per position in the cohort of players examined, whilst the position differences in various HRV parameters are a novel finding. The results are useful for understanding the somatic and fitness requirements of Czech professional soccer players that can be used for planning training and improving performance.

\section{Acknowledgment}

This study was supported by the university research grant IGA_FTK_2015_006.

\section{Conflict of interest}

There were no conflicts of interest.

\section{References}

Akselrod, S., Gordon, D., Ubel, F. A., Shannon, D. C., Berger, A. C., \& Cohen, R. J. (1981). Power spectrum analysis of heart rate fluctuation: A quantitative probe of beat-to-beat cardiovascular control. Science, 213, 220-222.

Atlaoui, D., Pichot, V., Lacoste, L., Barale, F., Lacour, J. R., \& Chatard, J. C. (2007). Heart rate variability, training variation and performance in elite swimmers. International Journal of Sports Medicine, 28, 394-400.

Aubert, A. E., Seps, B., \& Beckers, F. (2003). Heart rate variability in athletes. Sports Medicine, 33, 889-919.

Borresen, J., \& Lambert, M. I. (2008). Autonomic control of heart rate during and after exercise. Sports Medicine, 38, 633-646.

Bosquet, L. A., Merkari, S., Arvisais, D., \& Aubert, A. E. (2008). Is heart rate a convenient tool to monitor overreaching? A systematic review of the literature. British Journal of Sports Medicine, 42, 709-714.

Botek, M., Krejčí, J., Neuls, F., \& Novotný, J. (2013). Effect of modified method of autonomic nervous system activity assessment on results of heart rate variability analysis. Acta Universitatis Palackianae Olomucensis. Gymnica, 43(2), 39-46.

Botek, M., Krejčí, J., \& Weisser, R. (2014). Autonomic cardiac regulation and morpho-physiological responses to eight week training preparation in junior soccer players. Acta Gymnica, 44, 155-163.

Botek, M., McKune, A. J., Krejčí, J., Stejskal, P., \& Gába, A. (2014). Change in performance in response to training load adjustment based on autonomic activity. International Journal of Sports Medicine, 35, 482-488.

Buchheit, M. (2014). Monitoring training status with HR measures: Do all roads lead to Rome? Frontiers in Physiology, 27, 73 .
Buchheit, M., Chivot, A., Parouty, J., Mercier, D., Al Haddad, H., Laursen, P. B., \& Ahmaidi, S. (2010). Monitoring endurance running performance using cardiac parasympathetic function. European Journal of Applied Physiology, 108, 1153-1167.

Buchheit, M., Millet, G. P., Parisy, A., Pourchez, S., Laursen, P. B., \& Ahmaidi, S. (2008). Supramaximal training and postexercise parasympathetic reactivation in adolescents. Medicine \& Science in Sports \& Exercise, 40, 362-371.

Carling, C., \& Orhant, E. (2010). Variation in body composition in professional soccer players: Interseasonal and intraseasonal changes and the effects of exposure time and player position. Journal of Strength \& Conditioning Research, 24, 1332-1339.

Carter, J. B., Banister, E. W., \& Blaber, A. P. (2003). The effect of age on heart rate variability after endurance training. Medicine \& Science in Sport \& Exercise, 35, 1333-1340.

Casajus, J. A. (2001). Seasonal variation in fitness variables in professional soccer players. Journal of Sports Medicine and Physical Fitness, 41, 463-469.

Di Salvo, V., Baron, R., Tschan, H., Calderon Montero, F. J., Bachl, N., \& Pigozzi, F. (2007). Performance characteristics according to playing position in elite soccer. International Journal of Sports Medicine, 28, 222-227.

dos Santos, R. R. C., de Sousa, C. V., Olher, R. R., de Sousa Neto, I. V., Pereira, L. A., \& Sales, M. M. (2014). Comparison of the anaerobic power of Brazilian professional football players grouped by tactical position. Montenegrin Journal of Sports Science and Medicine, 3(2), 13-18.

Eckberg, D. L. (1997). Sympathovagal balance: A critical appraisal. Circulation, 96, 3224-3232.

Ekblom, B. (1986). Applied physiology of soccer. Sports Medicine, 3, 50-60.

Gil, S. M., Gil, J., Ruiz, F., Irazusta, A., \& Irazusta, J. (2007). Physiological and anthropometric characteristics of young soccer players according to their playing position: Relevance for the selection process. Journal of Strength and Conditioning Research, 21, 438-445.

Gil, S., Ruiz, F., Irazusta, A., Gil, J., \& Irazusta, J. (2007). Selection of young soccer players in terms of anthropometric and physiological factors. Journal of Sports Medicine and Physical Fitness, 47, 25-32.

Hautala, A. J., Kiviniemi, A. M., \& Tulppo, M. P. (2009). Individual responses to aerobic exercise: The role of the autonomic nervous system. Neuroscience \& Biobehavioral Reviews, 33, 107-115.

Hautala, A., Tulppo, M. P., Mäkikallio, T. H., Laukkanen, R., Nissilä, S., \& Huikuri, H. V. (2001). Changes in cardiac autonomic regulation after prolonged maximal exercise. Clinical Physiology, 21, 238-245.

Hedelin, R., Bjerle, P., \& Henriksson-Larsén, K. (2001). Heart rate variability in athletes: Relationship with central and peripheral performance. Medicine \& Science in Sports \& Exercise, 33, 1394-1398.

Hedelin, R., Wiklund, U., Bjerle, P., \& Henriksson-Larsén, K. (2000). Cardiac autonomic imbalance in an overtrained athlete. Medicine \& Science in Sports \& Exercise, 32, 1531-1533.

Helgerud, J., Rodas, G., Kemi, O. J., \& Hoff, J. (2011). Strength and endurance in elite football players. International Journal of Sports Medicine, 32, 677-682. 
Joksimović, A., Smajić, M., Molnar, S., \& Stanković, D. (2009). An analysis of anthropomorphological characteristics of participants in the 2008 European Football Championship. Serbian Journal of Sports Sciences, 3, 71-79.

Keogh, J. (1999). The use of physical fitness scores and anthropometric data to predict selection in an elite under 18 Australian rules football team. Journal of Science and Medicine in Sport, 2, 125-133.

Kouidi, E., Haritonidis, K., Koutlianos, N., \& Deligiannis, A. (2002). Effects of athletic training on heart rate variability triangular index. Clinical Physiology and Functional Imaging, 22, 279-284.

Lamberts, R. P., \& Lambert, M. I. (2009). Day-to-day variation in heart rate at different levels of submaximal exertion: Implications for monitoring training. Journal of Strength \& Conditioning Research, 23, 1005-1010.

Lamberts, R. P., Swart, J., Capostagno, B., Noakes, T. D., \& Lambert, M. I. (2010). Heart rate recovery as a guide to monitor fatigue and predict changes in performance parameters. Scandinavian Journal of Medicine \& Science in Sports, 20, 449-457.

Le Gall, F., Carling, C., Williams, M., \& Reilly, T. (2010). Anthropometric and fitness characteristics of international, professional and amateur male graduate soccer players from an elite youth academy. Journal of Science and Medicine in Sport, 13, 90-95.

MacArthur, D. G., \& North, K. N. (2005). Genes and human elite athletic performance. Human Genetics, 116, 331-339.

Magalhães Sales M., Vieira Browne, R. A., Asano, R. Y., dos Reis Vieira Olher, R., Vila Nova de Moraes, J. F., \& Simões, H. G. (2014). Physical fitness and anthropometric characteristics in professional soccer players of the United Arab Emirates. Revista Andaluza de Medicina del Deporte, 7, 106-110.

Malliani, A., Pagani, M., Lombardi, F., \& Cerutti, S. (1991). Cardiovascular neural regulation explored in the frequency domain. Circulation, 84, 482-492.

Matković, B., Janković, S., Ružić, L., \& Leko, G. (2003). Morphological characteristics of elite Croatian soccer players according to the team position. In The $5^{\text {th }}$ World Congress on Science and Football: Abstracts (pp. 172-173). Lisbon, Portugal: Editorial Gymnos.

Michailidis, Y., Michailidis, C., Primpa, E., Fatouros, I. G., Margonis, K., Chatzinikolaou, A., \& Methenitis, S. (2013). Intraseasonal change of body composition in professional soccer players and differences according to playing positions. Serbian Journal of Sports Sciences, 7, 39-44.

Milsom, J., Naughton, R., O’Boyle, A., Iqbal, Z., Morgans, R., Drust, B., \& Morton, J. P. (2015). Body composition assessment of English Premier League soccer players: A comparative DXA analysis of first team, U21 and U18 squads. Journal of Sports Sciences, 33, 1799-1806.

Naranjo Orellana, J., de la Cruz Torres, B., Sarabia Cachadiña, E, de Hoyo, M., \& Domínguez Cobo, S. (2015). Two new indexes for the assessment of autonomic balance in elite soccer players. International Journal of Sports Physiology and Performance, 10, 452-457.

Ori, Z., Monir, G., Weiss, J., Sayhouni, X., \& Singer, D. H. (1992). Heart rate variability. Frequency domain analysis. Cardiology Clinics, 10, 499-537.

Penttilä, J., Helminen, A., Jartti, T., Kuusela, T., Huikuri, H. V., Tulppo, M. P., \& Scheinin, H. (2001). Time domain, geometrical and frequency domain analysis of cardiac vagal outflow: Effects of various respiratory patterns. Clinical Physiology, 21, 365-376.

Pichot, V., Roche, F., Gaspoz, J. M., Enjolras, F., Antoniadis, A., Minini, P., \& Barthelemy, J. C. (2000). Relation between heart rate variability and training load in middle distance runners. Medicine \& Science in Sports \& Exercise, 32, 1729-1736.

Rampinini, E., Sassi, A., Sassi, R., \& Impellizzeri, F. M. (2004, April). Variables influencing fatigue in soccer performance. Paper presented at the XIII International Congress on Sport Rehabilitation and Traumatology, Milan, Italy.

Reilly, T. (1996). Fitness assessment. In T. Reilly (Ed.), Science and soccer (pp. 25-50). London, United Kingdom: E \& FN Spon.

Reilly, T., Bangsbo, J., \& Franks, A. (2000). Anthropometric and physiological predispositions for elite soccer. Journal of Sports Sciences, 18, 669-683.

Shephard, R. J., \& Åstrand, P. O. (Eds.). (1992). Endurance in sport. Oxford, United Kingdom: Blackwell Science.

Silvestre, R., West, C., Maresh, C. M., \& Kraemer, W. J. (2006). Body composition and physical performance in men's soccer: A study of a national collegiate athletic association division team. Journal of Strength \& Conditioning Research, 20, 177-183.

Stølen, T., Chamari, K., Castagna, C., \& Wisløff, U. (2005). Physiology of soccer: An update. Sports Medicine, 35, 501-536.

Strøyer, J., Hansen, L., \& Klausen, K. (2004). Physiological profile and activity pattern of young soccer players during match play. Medicine \& Science in Sports \& Exercise, 36, 168-174.

Sutton, L., Scott, M., Wallace, J., \& Reilly, T. (2009). Body composition of English Premier League soccer players: Influence of playing position, international status, and ethnicity. Journal of Sports Sciences, 27, 1019-1026.

Task Force of the European Society of Cardiology and the North American Society of Pacing and Electrophysiology. (1996). Heart rate variability: Standards of measurement, physiological interpretation and clinical use. Circulation, 93, 1043-1065.

Wilson, J., \& O’Donoghue, P. G. (2005). Physiological profiling of Northern Ireland amateur league soccer players. Journal of Sports Sciences, 23, 126.

Wisløff, U., Helgerud, J., \& Hoff, J. (1998). Strength and endurance of elite soccer players. Medicine \& Science in Sports \& Exercise, 30, 462-467. 